\title{
Synthesis and characterization of core-shell gold nanoparticles with poly(vinyl pyrrolidone) from a new precursor salt
}

\author{
M. Behera $\cdot$ S. Ram
}

Received: 6 February 2012/Accepted: 25 February 2012/Published online: 7 March 2012

(c) The Author(s) 2012. This article is published with open access at Springerlink.com

\begin{abstract}
In this article, we report a facile one-step chemical synthesis of gold ( $\mathrm{Au}$ ) nanoparticles (GNPs) from a new precursor salt i.e., gold hydroxide in the presence of poly(vinyl pyrrolidone) (PVP) polymer. The non-aqueous dispersion of GNPs was comprehensively characterized by UV-Visible, FTIR, zeta potential, and transmission electron microscope (TEM). A strong surface plasmon resonance band at $529 \mathrm{~nm}$ in the UV-Visible spectrum confirms the formation of GNPs in the Au colloid. The FTIR spectroscopic results showed that PVP molecules get chemisorbed onto the surface of GNP via O-atom of carbonyl group. A negative zeta potential of $(-) 16 \mathrm{mV}$ reveals accumulation of nonbonding electrons of O-atom of carbonyl group of PVP molecules on the nanosurface of GNP. TEM images demonstrate a core-shell nanostructure with an Au-crystalline core covered by a thin amorphous PVP-shell. PVP-capped GNPs could be a potential candidate for bio-sensing, catalysis, and other applications.
\end{abstract}

Keywords Gold nanoparticles · Core-shell nanostructure · Zeta potential $\cdot$ Chemisorption

\section{Introduction}

Noble metal nanoparticles (NPs) have attracted a significant interest in materials and biological sciences owing to

\section{Behera ( $\square)$}

Silicon Institute of Technology, Silicon Hills,

Bhubaneswar 751024, India

e-mail: mano.silicon@gmail.com

\section{S. Ram}

Materials Science Centre, Indian Institute of Technology, Kharagpur 721302, India their potential applications in catalysis (Eustis and El-Sayed 2006; Tang et al. 2008; Daniel and Astruc 2004), biosensors (Eustis and El-Sayed 2006; Tang et al. 2008; Daniel and Astruc 2004), biomedicals (Eustis and El-Sayed 2006; Daniel and Astruc 2004; Ringe et al. 2010), photonics (Murphy et al. 2005) and heat transfer (Shalklvicht et al. 2010; Zhang et al. 2010). In recent years, gold nanoparticles (GNPs) of various sizes and shapes have received much attention from both scientists and academia because of their unique physical, chemical, photochemical, opto-electronic, and biomedical properties (Eustis and El-Sayed 2006; Tang et al. 2008; Daniel and Astruc 2004; Ringe et al. 2010; Murphy et al. 2005). The brilliant ruby color seen in stained glass windows is attributed to the presence of GNPs (Faraday 1857). Such bright color is due to collective oscillation of the electrons in the conduction band, known as surface plasmon oscillation. As the frequency of such oscillation lies in the visible region of the spectrum in gold, it gives rise to a strong surface plasmon resonance (SPR) absorption (Eustis and El-Sayed 2006; Ringe et al. 2010). Such absorption is absent in bulk metal and occurs only in nanoscale metal NPs when the incident photon frequency resonates the collective oscillation of the conduction electrons. Because of larger absorption coefficient of GNP than dye molecule, SPR is a powerful tool for biological sensors, surface-enhanced Raman spectroscopy, and many optical devices (Eustis and El-Sayed 2006; Tang et al. 2008; Daniel and Astruc 2004; Ringe et al. 2010; Murphy et al. 2005). As SPR is strongly influenced by size, shape, aggregation, composition, and the local dielectric environment of the NP (Ringe et al. 2010; Kelly et al. 2003), it can be tuned by proper selection of a synthetic route and using an efficient capping agent. Due to high surface area and surface activity, GNPs show a great tendency toward aggregation. Such agglomeration process is 
not required for all mentioned applications of GNPs. In this regard, a chemical method is way ahead of other synthetic routes, as it is simple, uses a weak reducing agent, and employs an efficient capping agent like poly(vinyl pyrrolidone) PVP to stop the growth process at nanoscopic level and foils aggregation of GNPs in a colloidal solution (Seo et al. 2006; Mishra et al. 2009; Ram and Fecht 2011).

Poly(vinyl pyrrolidone), an amphiphilic polymer, is a widely used encapsulating agent in the synthesis of various architectures of metal NPs (Seo et al. 2006; Mishra et al. 2009; Ram and Fecht 2011). It is not only used as a reducing/nucleating agent but also as an excellent shape and size regulating agent (Tang et al. 2008; Ringe et al. 2010; Seo et al. 2006; Mishra et al. 2009; Ram and Fecht 2011). As PVP is non-toxic and biodegradable, it possesses excellent biomedical properties (Seo et al. 2006; Mishra et al. 2009; Ram and Fecht 2011; Kamada et al. 2003) and helps in decreasing the toxicity of NPs by providing an inert coating over it. In the molecular structure of PVP, the polyvinyl backbone serves as a tail group (hydrophobic), whereas the pyrrolidone group serves as a head group (hydrophilic). The head carbonyl $(\mathrm{C}=\mathrm{O})$ groups of PVP can interact with surfaces of GNP, whereas the long tail groups can adjust the distances among adsorbed head groups to provide colloidal stability by steric effect (Seo et al. 2006; Mishra et al. 2009; Ram and Fecht 2011). Upon mesomerism in the pyrrolidone ring in the presence of a hydrogen donor (Rothschild 1972), PVP becomes reactive to build up charge-transfer complexes via O-atom with electron acceptors like fullerenes $\left(\mathrm{C}_{60} / \mathrm{C}_{70}\right)$ (Khairullin et al. 1997 Behera and Ram 2012) and metal NPs (e.g., Au, Ag, Pt, Rh, etc.) (Seo et al. 2006; Mishra et al. 2009; Ram and Fecht 2011; Borodko et al. 2006, 2007).

In this work, a novel ultrasonic-aided one-step chemical synthesis of core-shell GNPs in the presence of PVP molecules in an alcoholic medium (i.e., in 1-butanol) was reported. Gold hydroxide $\mathrm{Au}(\mathrm{OH})_{3}$, a new precursor salt, tested in this work instead of widely used hydrated tetrachloro auric acid $\left(\mathrm{HAuCl}_{4} \cdot 3 \mathrm{H}_{2} \mathrm{O}\right)$ salt for synthesis of GNPs (Tang et al. 2008; Ringe et al. 2010, Mishra et al. 2009 Ram and Fecht 2011). Samples were studied in terms of UV-Visible, Fourier transform infrared (FTIR), Zeta potential, and transmission electron microscope (TEM).

\section{Materials and methods}

Synthesis of core-shell Au-NPs

All glasswares used for the synthesis of GNPs were thoroughly cleaned in an ultrasonic bath and oven-dried prior to use. Reagent grade $\mathrm{Au}(\mathrm{OH})_{3}$ powder was purchased from Alfa Aesar and was dissolved in dilute $\mathrm{HNO}_{3}$ to prepare a stock solution of $0.05 \mathrm{M} \mathrm{Au}\left(\mathrm{NO}_{3}\right)_{3}$. PVP $(25 \mathrm{kDa})$ and 1-butanol were purchased from Sigma Aldrich and Merck, respectively. The chemicals were used as received without further purification. At first, an alcoholic mother solution of PVP (40 g/L) was prepared in 1-butanol by mechanical stirring for $3 \mathrm{~h}$ at $60-70^{\circ} \mathrm{C}$. After this, a specific volume of gold nitrate solution was added dropwise during stirring to a $5 \mathrm{~mL}$ of $40 \mathrm{~g} / \mathrm{L} \mathrm{PVP} \mathrm{solution}$ in 1-butanol at room temperature. After magnetic stirring for $5 \mathrm{~min}$, the solution was then sonicated at $50^{\circ} \mathrm{C}$ in an ultrasonicator (OSCAR, $20 \mathrm{kHz}$ frequency and $250 \mathrm{~W}$ power) for $5 \mathrm{~min}$ so that a light purple colored nanocolloids consisting of $2 \mu \mathrm{M}$ GNPs with $40 \mathrm{~g} / \mathrm{L}$ PVP molecules were obtained in 1-butanol. Stable NF obtained was studied in terms of the UV-Visible, FTIR, Zeta potential, and microstructure.

\section{Characterization}

The UV-Visible spectra of non-aqueous dispersion of GNPs with and without PVP molecules were measured under identical conditions on a Perkin Elmer double beam spectrophotometer (LAMBDA 1050) at $26^{\circ} \mathrm{C}$. The sample was filled in a conventional quartz cell of $10-\mathrm{mm}$ optical length and the spectra were recorded against a reference (1-butanol with $40 \mathrm{~g} / \mathrm{L}$ PVP, or 1-butanol) in an identical cell. FTIR spectra were recorded on a Thermo Nicolet Corporation FTIR Spectrometer (Model NEXUS-870) in an attenuated total reflectance mode using a $\mathrm{ZnSe}$ crystal as a sample holder.

Zeta potential $(\xi)$ and surface conductivity $\left(\sigma_{\mathrm{sc}}\right)$ were measured using a Malvern Nano ZS instrument using phase analysis light scattering technique. Diluted samples were sonicated for $60 \mathrm{~s}$ prior to measurements. Microscopic image of PVP-encapsulated Au-NPs was studied with a high resolution transmission electron microscope (HRTEM) of JEM2100 (JEOL, Japan) operated at an accelerating voltage of $100 \mathrm{kV}$. A $50-\mu \mathrm{L}$ colloidal gold solution was dropped on a carbon-coated 400-mesh copper grid. The grid was then dried in desiccators by keeping overnight at room temperature.

\section{Results and discussion}

\section{UV-Visible and FTIR spectra}

UV-Visible is a powerful spectral technique used to confirm the formation of GNPs in a colloidal solution. Figure 1 shows the absorption spectra $(300-750 \mathrm{~nm})$ obtained from (a) $40 \mathrm{~g} / \mathrm{L}$ PVP base solution and (b) base solution with $2 \mu \mathrm{M}$ GNPs in 1-butanol. The photographs taken of respective samples were shown as inset. A colorless sample (a) turns light purple color in the presence of $2 \mu \mathrm{M}$ GNPs. 
A broad surface plasmon resonance absorption band at $529 \mathrm{~nm}$ obtained from collective oscillation of valence electrons further confirms the presence of 10 - to $15-\mathrm{nm}$ diameter spherical GNPs in the NF. This result is well supported by TEM image shown in Fig. 4a. The pure PVP solution is found to be totally transparent in the visible region as seen from Fig. 1. Again, absence of a CT band near $320 \mathrm{~nm}$ which is attributed to unreduced gold $\left(\mathrm{Au}^{3+}\right)$ ions reveals complete reduction of $\mathrm{Au}^{3+}$ ions to $\mathrm{Au}^{0}$ (i.e., GNPs) in the reaction medium (Sakai and Alexandridis 2005; Gangopadhayay and Chakravorty 1961).

In order to validate whether the PVP molecules have been adhered to the surface of GNPs, the vibrational spectra (as shown in Fig. 2) of Au nanocolloids and mother PVP solution were recorded on a FTIR instrument. As seen from the spectra, some characteristic vibrational bands of PVP molecule change when the PVP molecules were adsorbed on the surface of GNP. A noticeable augmentation in the band intensities in $\mathrm{C}-\mathrm{H}(2,961,2,936$, and $\left.2,872 \mathrm{~cm}^{-1}\right)$ stretching, $\mathrm{C}-\mathrm{N}\left(1,463 \mathrm{~cm}^{-1}\right)$ stretching and $\mathrm{CH}_{2}\left(1,381 \mathrm{~cm}^{-1}\right)$ bending vibrations in the pyrrolidone ring of PVP molecules was observed from the spectrum of sample (b) consisting of $2 \mu \mathrm{M}$ GNPs with $40 \mathrm{~g} / \mathrm{L}$ PVP. This occurs only when PVP molecules get adsorbed on the nanosurface of Au-NP via n-electron of O-atom of lactam unit. A similarly enhanced intensity in these modes was observed when PVP molecules get coated on the surface of fullerene (Behera and Ram 2012). Moreover Borodko et al. (2006, 2007) have observed similarly enhanced intensities in these modes but in PVP-encapsulated Pt and Rh nanocrystals. Further, a slight red-shift in the $\mathrm{C}=\mathrm{O}$ stretching vibration from 1,667 to $1,661 \mathrm{~cm}^{-1}$ in the presence of GNPs reveals formation of a weak donor-acceptor type interaction by transfer of nonbonding electron of O-atom of PVP molecules to Au surface (Sakai and Alexandridis 2005; Bai et al. 2007).

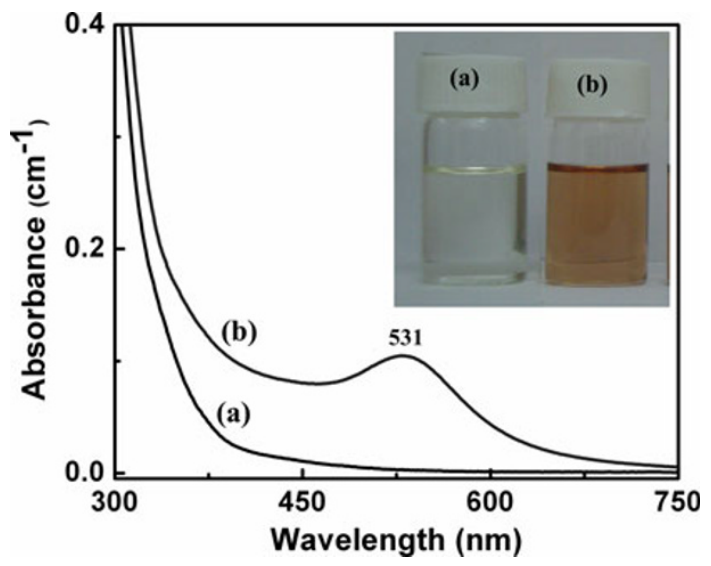

Fig. 1 Absorption spectra of $40 \mathrm{~g} / \mathrm{L}$ PVP molecules consisting of $a 0$, and $b 2 \mu \mathrm{M}$ GNPs in 1-butanol. The photographs taken of respective samples were shown as inset

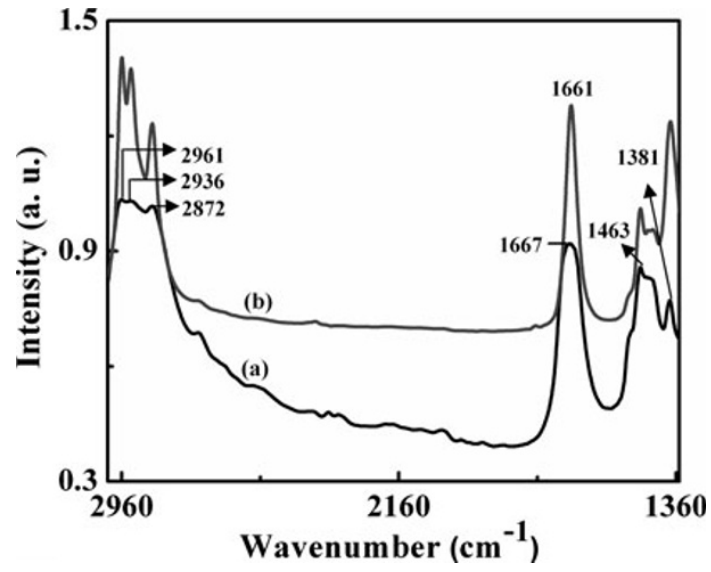

Fig. 2 Vibrational spectra of $40 \mathrm{~g} / \mathrm{L}$ PVP molecules consisting of $a 0$, and $b 2 \mu \mathrm{M}$ GNPs in 1-butanol

Zeta potential measurement

Surface charge on GNPs in the colloids was studied in terms of zeta potential $(\xi)$ measurement. Such studies help in understanding the stabilization mechanism of Au colloids in a particular medium. As shown in Fig. 3, a mother solution (a) of $40 \mathrm{~g} / \mathrm{L}$ PVP displays a prominent zeta-band at $(-) 6.0 \mathrm{mV}$ with surface conductivity $\left(\sigma_{\mathrm{sc}}\right)=0.014 \mathrm{mS} / \mathrm{cm}$ at $7.5 \mathrm{pH}$. Whereas, a nanocolloids (b) consisting of $2 \mu \mathrm{M}$ GNPs with $40 \mathrm{~g} / \mathrm{L}$ PVP result in larger $\xi$-value of $(-) 16 \mathrm{mV}$, with an enhanced $\sigma_{\mathrm{sc}}=0.13 \mathrm{mS} / \mathrm{cm}$. A larger $\xi$-value with an enhanced $\sigma_{\mathrm{sc}}$-value is a result of an effectively negative charge confined on a nanosurface of GNP in assistance of surface PVP layer (Sakai and Alexandridis 2005; Bianca et al. 2000). A transfer of $\mathrm{C}=\mathrm{O}$ n-electrons from a nucleophilic PVP molecule to an electrophilic $\mathrm{C}_{60}$ molecule shares the localized surface charge density. When PVP-capped GNPs approach toward each other, the negatively charged surface of GNPs prevents

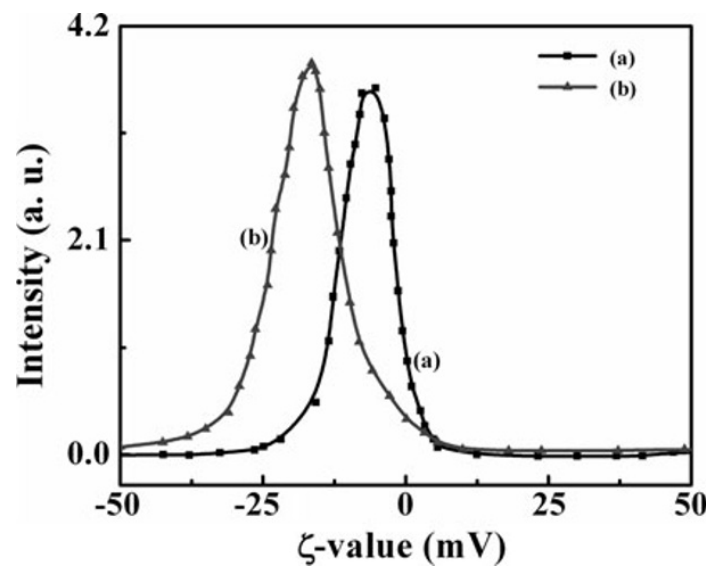

Fig. $3 \xi$-bands profile of $40 \mathrm{~g} / \mathrm{L}$ PVP molecules consisting of $a 0$, and $b 2 \mu \mathrm{M}$ GNPs in 1-butanol at $\mathrm{pH}=7.5$ 


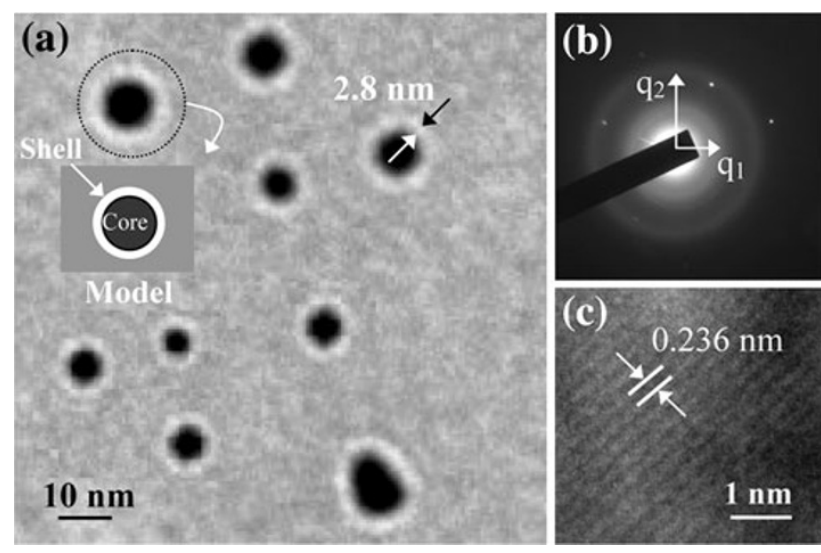

Fig. 4 TEM images taken from $2 \mu \mathrm{M}$ GNPs with $4 \mathrm{~g} / \mathrm{L}$ PVP molecules in 1-butanol. A typical SAED pattern shows two diffraction halos obtained from the amorphous surface layer and lattice fringes confirms crystalline nature of Au core in a core-shell structure of PVP-encapsulated GNP are placed as insets. A model core-shell structure drawn and affixed as inset in Fig. 4a in support of images

flocculation and hence stabilizes the colloids by electrostatic repulsion and steric effect.

\section{Microstructural investigation}

Direct information about the shape, size, and distribution of PVP-encapsulated GNPs can be studied from the TEM image. Figure 4a depicts a TEM image obtained from $2 \mu \mathrm{M}$ Au-NPs with $4 \mathrm{~g} / \mathrm{L}$ PVP. Mostly, spherical GNPs of average $10-15 \mathrm{~nm}$ diameter are seen in this image. The selected area electron diffraction (SAED) pattern (Fig. 4b) from the surface of PVP-encapsulated GNP illustrates an amorphous surface structure of modified GNPs with two distinct halos $q_{1}$ and $q_{2}$ of $d_{h k l}$ values (inter-planar spacing) $0.224 \mathrm{~nm}$ corresponding to (111) and $0.139 \mathrm{~nm}$ corresponding to (220). Our group (Mishra et al. 2009) has already reported similar halos obtained from the surface of PVP-modified GNP. A high resolution TEM image (4c) shows lattice fringes with inter-planar spacing $0.236 \mathrm{~nm}$ corresponds to (111) reflection of Au nanocrystal. Moreover, TEM image demonstrates a core-shell nanostructure of Au-crystalline core covered by a $2.8 \mathrm{~nm}$ shell of amorphous PVP layer with whitish contrast. A similar type of core-shell structure is reported in aqueous colloids of GNPs with PVP molecules (Ram and Fecht 2011). In support of TEM images, a model core-shell structure was drawn and affixed as inset in Fig. 4a.

\section{Conclusion}

Nearly monodisperse spherical GNPs were synthesized successfully from a gold hydroxide $\mathrm{Au}(\mathrm{OH})_{3}$ precursor salt in the presence of PVP molecules in 1-butanol. The SPR band in UV-Visible spectrum of colloid confirms presence of GNPs in the non-aqueous system. The FTIR results indicate that PVP molecules get adsorbed on the surface of GNP via donor-acceptor type interaction between carbonyl group and GNP. A nonbonding electron transfer from O-atom of carbonyl group of PVP molecules to the surface of GNP results in a negatively charged Au surface with a zeta potential of $(-) 16 \mathrm{mV}$. TEM image shows formation of nearly monodisperse polymer-capped GNPs with a core-shell structure. Monodisperse PVP-capped GNPs could be a potential candidate for catalytic applications.

Acknowledgments This work has been supported by Silicon Institute of Technology, Silicon Hills, Bhubaneswar, India.

Open Access This article is distributed under the terms of the Creative Commons Attribution License which permits any use, distribution, and reproduction in any medium, provided the original author(s) and the source are credited.

\section{References}

Bai J, Li Y, Du J, Wang S, Zheng J, Yang Q, Chen X (2007) One pot synthesis of polyacrylamide-gold nanocomposite. Mat Chem Phys 106:412-415

Behera M, Ram S (2012) Solubilization and stabilization of fullerene $\mathrm{C}_{60}$ in presence of poly(vinyl pyrrolidone) molecules in water. J Incl Phenom Macrocyl Chem 72:233-239

Bianca MI, Jan KGD, Marcel RB, Albert PP (2000) Colloidal dispersion of gold rods characterized by dynamic light scattering and electrophoresis. Langmuir 16:459-464

Borodko Y, Habas SE, Koebel M, Yang P, Frei H, Somorjai GA (2006) Probing the interaction of poly(vinylpyrrolidone) with platinum nanocrystals by UV-Raman and FTIR. J Phys Chem B 110:23052-23059

Borodko Y, Humphrey SM, Tilley TD, Frei H, Somorjai GA (2007) Charge-transfer interaction of poly(vinylpyrrolidone) with platinum and rhodium nanoparticles. J Phys Chem C 111:6288-6295

Daniel MC, Astruc D (2004) Gold nanoparticles: assembly, supramolecular chemistry, quantum-sized-related properties, and applications toward biology, catalysis, and nanotechnology. Chem Rev 104:293-346

Eustis S, El-Sayed MA (2006) Why gold nanoparticles are more precious than pretty gold: noble metal surface plasmon resonance and its enhancement of the radiative and nonradiative properties of nanocrystals of different shapes. Chem Soc Rev 35:209-217

Faraday M (1857) Experimental relations of gold (and other metals). Philos Trans Royal Soc London 147:145-181

Gangopadhayay AK, Chakravorty A (1961) Charge transfer spectra of some gold (III) complexes. J Chem Phys 35:2206-2209

Kamada H, Tsutsumi Y, Kamada KS, Yamamoto Y, Yoshioka Y, Okamoto T, Nakagawa S, Nagata S, Mayumi T (2003) Synthesis of a poly(vinylpyrrolidone-co-dimethyl maleic anhydride) co-polymer and its application for renal drug targeting. Nat Biotechnol 21:399-404

Kelly KL, Coronado E, Zhao LL, Schatz GC (2003) The optical properties of metal nanoparticles: the influence of size, shape, and dielectric environment. J Phys Chem B 107:668-677 
Khairullin II, Chen Y, Hwang L (1997) Evidence for electron charge transfer in the polyvinylpyrrolidone- $\mathrm{C}_{60}$ system as seen from ESR spectra. Chem Phys Lett 275:1-6

Mishra A, Ram S, Ghosh G (2009) Dynamic light scattering and optical absorption in biological nanofluids of gold nanoparticles in poly(vinyl pyrrolidone) molecules. J Phys Chem C 113: 6976-6982

Murphy CJ, Sau TK, Gole AM, Orendorff CJ, Gao J, Guo L, Hyunadi SE, Li T (2005) Anisotropic metal nanoparticles: synthesis, assembly, and optical applications. J Phys Chem B 109:1385713870

Ram S, Fecht HJ (2011) Modulating up-energy transfer and violetblue light emission in gold nanoparticles with surface adsorption of poly(vinyl pyrrolidone) molecules. J Phys Chem C 115:78177828

Ringe E, McMahon JM, Sohn K, Cobley C, Xia Y, Huang J, Schatz GC, Marks LD, Duyne RV (2010) Unravelling the effects of size, composition, and substrate on the localized surface plasmon resonance frequencies of gold and silver nanocubes: a systematic single-particle approach. J Phys Chem C 114:12511-12516

Rothschild WG (1972) Binding of hydrogen donors by peptide groups of lactams. Identity of the reaction sites. J Am Chem Soc 94:8676-8683
Sakai T, Alexandridis P (2005) Size- and shape-controlled synthesis of colloidal gold through autoreduction of the auric cation by poly(ethylene-oxide)-poly(propylene oxide) block copolymers in aqueous solutions at ambient conditions. Nanotechnology 16:S334

Seo D, Park JC, Song H (2006) Polyhedral gold nanocrystals with Oh symmetry: from octahedral to cubes. J Am Chem Soc 128: 14863-14870

Shalklvicht N, Escher W, Burgi T, Michel B, Si-Ahmed L, Poulikakos D (2010) On the thermal conductivity of gold nanoparticle colloids. Langmuir 26:663-670

Tang XL, Jiang P, Ge GL, Tsuji M, Xie SS, Guo YJ (2008) Poly ( $N$-vinyl-2-pyrrolidone) (PVP)-capped dendritic gold nanoparticles by a one-step hydrothermal route and their high SERS effect. Langmuir 24:1763-1768

Zhang H, Wu Q, Lin J, Chen J, Xu Z (2010) Thermal conductivity of polyethylene glycol nanofluids containing carbon coated metal nanoparticles. J Appl Phys 108:124304-124309 